\title{
Planck scale potential associated with particles
}

\author{
D. L. Bulathsinghala, K. A. I. L. Wijewardena Gamalath* \\ Department of Physics, University of Colombo, Colombo 3, Sri Lanka \\ ${ }^{*}$ E-mail address: imalie@phys.cmb.ac.lk
}

\begin{abstract}
As the particles originating from point-like entities are associated with infinite self energies, a postulate, that the scalar-potential associated with particles are bounded by a Planck scale potential is introduced. By defining the self energy of a particle, equivalences between charge-energy and massenergy are obtained. The electromagnetic energy-momentum equation, de-Broglie's electromagnetic wave-length and frequency for a charge particle in motion are presented resolving the " $4 / 3$ " discrepancy. The non-covariance nature of the present classical electrodynamics is discussed and how the proposed postulate makes it a fully covariant theorem with the rest of the classical electrodynamics is presented. A way electromagnetic energy-momentum equation could potentially resolve the stability-problem of a charge particle is discussed and thereby a theoretical explanation to electron's spin is presented.
\end{abstract}

Keywords: Planck scale potential; mass-energy equivalence; charge-energy equivalence; energymomentum relation; electromagnetic momentum; de-Broglie's wave length

\section{INTRODUCTION}

In classical electrostatics, the self energy of a particle of charge assumed to be uniformly distributed over its surface, constructed by bringing in infinitesimal amounts of charge quantities of the particle from infinity, diverges or goes to infinity, when the radius approaches to zero. However to avoid the infinite energy problem, if the charge particle is assumed to be associated with a finite radius, then it give rise to the problem of explaining how the like-charge distribution of the particle is held together against the repulsive nature of the like-charges, known as the "stability-problem".

Historically, a few different approaches have been proposed in order to account for the self-energy problem arising from the point-like charge particles and the stability-problem arising from the finite radius models. Stokes [1] showed in 1844 that the inertia of a body moving in an incompressible perfect fluid is increased.

Noticing that electromagnetic momentum and energy of charged bodies and therefore their masses depend on the speed of the bodies, Thomson in 1881 [2] recognized this effect for moving charged particles and showed that it is harder to set in motion a charged sphere moving in a space filled with a medium of a specific inductive capacity than an uncharged body. Due to this self-induction effect, electrostatic energy behaves as having some sort of momentum and apparent electromagnetic mass, which can increase the ordinary mechanical mass of the bodies, or in modern terms, the increase should arise from their electromagnetic self energy. 
Using this model Searle [3] in 1897, derived the electromagnetic energy of a moving charge spheroid shell. In 1904, Lorentz [4] computed the electromagnetic momentum for a moving charge spheroid shell. However, the electromagnetic energy and momentum they obtained were neither relativistically covariant nor transformed as an energy-momentum four vector. In order to remove the 4/3 discrepancy appearing, Poincare [5] postulated that a nonelectromagnetic force was necessary to hold the like-charge distribution, and by doing so, he was able to solve the stability and the discrepancy problems.

However, the non-covariant ad-hoc forces associated with Poincare's postulate are assumed to compensate for the non-covariance of the electromagnetic force so that the entire electron system becomes covariant.

Thereafter many others attempted to resolve the 4/3 discrepancy and noteworthy among them are Fermi [6], Mandel [7], Wilson [8], Dirac [9], Pryce [10], Kwal [11] and Rohrlich [12]. By showing that the Trouton-Noble experiment's null result [13] can be explained if the energy density of an electromagnetic field can be expressed as the difference in electric and magnetic energy in vacuum rather than their addition, Butler [14] derived the energymomentum four vector and resolve the " $4 / 3$ " discrepancy problem and showed that the source of the non-covariance of the energy and momentum density expressions arise from the procedure used to derive the Poynting's theorem, which is covariant only in the absence of charges in moving frames.

Stratton has also pointed out that "the classical interpretation of Poynting's theorem appears to rest to a considerable degree on hypothesis" [15] while Pauli had stated that "the Maxwell-Lorentz electrodynamics is quite incompatible with the existence of charges, unless it is supplemented by extraneous theoretical concepts" [16]. A similar analysis on hidden momentum and electromagnetic mass of a charge body was carried out by Hnizdo [17].

In quantum electro-dynamics with its renormalization techniques, the energy associated with an electron is separated into two parts: the energy associated by its interactions with other charge particles and the self-energy associated by its interactions with itself. In renormalization, the part that interacts with itself is removed or taken out from the theory and therefore the electron's charge doesn't fly-off or repel itself.

With this treatment, the infinities which arise when the radius of the spherical electron goes to zero, is removed.

In the present paper, a new postulate, that a scalar potential associated with particles bounded by the Planck scale is presented. Including this postulate, equivalence between charge and energy was achieved and conventional mass-energy equivalence was re-affirmed by a similar procedure.

Deriving the relativistic energy-momentum relation for a charge particle in motion, both relativistically covariant, energy and momentum expressions were obtained resolving the $4 / 3$ discrepancy.

Using de-Broglie's hypothesis, an electromagnetic matter wavelength and matter frequency for a charge particle in motion are presented. With the proposed postulate the noncovariance nature of the present classical electrodynamics was changed into a fully covariant form. Discussing how the proposed postulate could potentially resolve the stability problem of a charge particle, a theoretical explanation to the electron's spin is presented. 


\section{SELF ENERGY OF A CHARGE PARTICLE}

The energy of a particle of charge $q$, assumed to be uniformly distributed over the surface of the particle's body of radius $r$, is given by:

$U_{0}=\frac{1}{2}\left(\frac{q^{2}}{4 \pi \varepsilon_{0} r}\right)$

Similarly, if the charge $q$ is assumed to be distributed over the particle's volume with a constant charge density, the energy expression reads:

$U_{0}=\frac{3}{5}\left(\frac{q^{2}}{4 \pi \varepsilon_{0} r}\right)$

The energy of the charge particle due to surface charge density or volume charge density diverges or said to give rise to a singularity, when the radius approaches to zero. In other words the corresponding energies become infinite, for point-like charge particles. Further, they only take into account the amount of potential energy that is stored in constructing such configurations, when bringing infinitesimal amounts of charge quantities from infinity, and that the subsequent infinitesimal charge amounts do not possess self energies associated to them. The conventional energy expressions given in equations 1 and 2 are based on the assumption that charge is continuous and indefinitely sub divisible and that the subdivided infinitesimal charge quantities alone do not possess a self energy associated to them. Thus, the expressions in equations 1 and 2 can be regarded as the potential energy of a system comprising infinite number of infinitesimal charge quantities, whose individual self energies are assumed to be zero.

Einstein [15] proposed equivalence between mass and energy in 1905 and concluded that the mass of a body is a measure of its energy content. That is, if the energy changes by $E$, the mass $m$ changes in the same sense, i.e.

$$
E=m c^{2}
$$

Further, the relativistic energy of a particle with rest mass $m$ and velocity $u$, is obtained from the energy momentum relation.

$$
E^{2}=(\gamma m u c)^{2}+\left(m c^{2}\right)^{2}
$$

Nevertheless, in the context of relativity, mass is not considered as an additive quantity, in the sense that, in a system, the collection of rest masses of the particles adding up to give the total rest mass of the system. Instead, the energy-momentum equation quantifies the amount of total invariant mass $M$ of the system:

$$
\left(M c^{2}\right)^{2}=\left(\sum_{i} E_{i}\right)^{2}-\left|\left(\sum_{i} \gamma_{i} m_{i} \boldsymbol{u}_{i} c\right)\right|^{2}
$$


where the speed $u_{i}$ of each particle $m_{i}$ are obtained relative to the center-of-momentum of the mass body $M$. A system composed of a collection of particles obeys the equation 5 , whereas individual particles at rest obey the equation 3 while when they are in a constant velocity motion they obey equation 4.

The mass-energy equivalence given in equation 5 is not constructed by bringing in an infinitesimal amount of mass quantities, each associated with zero self energy content at infinity and even at infinity, a corresponding infinitesimal amount of mass quantity $\delta m$, is assumed to be associated with a self energy:

$$
E=\delta m c^{2}
$$

In view of the self-energy problem arising from point-like charge particle models, the zero self-energies associated with infinitesimal amounts of charge quantities at infinity arising from finite radius charge particle models and the $4 / 3$ problem associated with the classical electron theory, the postulate that the scalar-potential associated with particles are bounded by the Planck scale potential is put forwarded to construct a physical theory consistent with the theory of relativity. The self-energy associated with a charge quantity $q$, interacting with its own field $\phi_{E}$ is given by:

$$
E=q \phi_{E}=q\left(\frac{q}{4 \pi \varepsilon_{0} r}\right)
$$

As the radius approaches to zero, the scalar-potential $\phi_{E}$ becomes infinite and thereby the corresponding self-energy of the charge particle becomes infinite as well. However from the proposed postulate, the scalar-potential associated with particles are bounded by the Planck scale potential or voltage $V_{\text {planck: }}$ :

$$
\phi_{E}=\left(\frac{q}{4 \pi \varepsilon_{0} r}\right)_{\text {planck }}=V_{\text {planck }}
$$

Therefore the self-energy of a charge particle is obtained as

$$
E=q V_{\text {planck }}
$$

\section{MASS-ENERGY EQUIVALENCE}

The self-energy associated with a spherical mass quantity $m$ with a radius $r$ interacting with its own field $\phi_{G}$ is given by:

$$
E=m \phi_{G}=m\left(\frac{G m}{r}\right)
$$


where $G$ is the universal gravitational constant. As the radius approaches to zero, the scalarpotential $\phi_{G}$ becomes infinite and thereby the corresponding self-energy of the mass particle becomes infinite as well. However, from the proposed postulate, the scalar-potential associated with the particles are bounded by the Planck scale potential such that:

$$
\phi_{G}=\left(\frac{G m}{r}\right)_{\text {planck }}=(\text { velocity })_{\text {planck }}^{2}=c^{2}
$$

Thus, the self-energy of a mass particle is obtained as equation 3 .

Historically, Poincare arrived at equation 3 in 1900 based on the concept of the radiation pressure, associated with electromagnetic radiation energy with a fictitious-fluid having mass and momentum. Einstein first entered the discussion in 1905 by relating the radiation energy to the change of mass [15]. He then affirmed this relation in many different presentations, revisiting the discussion with different experiments, devised to affirm the equivalence. However, from a recent review from Hecht [16], it is argued that Einstein was not able to provide a conclusive general proof of this seminal hypothesis from first principles.

\section{RELATIVISTIC ENERGY-MOMENTUM RELATION ASSOCIATED WITH A CHARGED PARTICLE IN MOTION}

Starting from the classical interpretation, a generalized relation for the change in energy $d E$ and momentum $d \boldsymbol{P}$ can be derived for a particle in motion, from force $\boldsymbol{F}$, and velocity $\boldsymbol{u}$. Consider a particle moving in $x$-direction under an external force $F$, the change in energy,

$d E=\boldsymbol{F} \cdot d \boldsymbol{r}=\frac{d \boldsymbol{p}}{d t} \cdot d \boldsymbol{r}=d \boldsymbol{p} \cdot \boldsymbol{u} \Rightarrow \frac{d E}{d p}=u$

Further, the electromagnetic vector-potential $\boldsymbol{A}$, located on the surface of a charge particle with charge $q$ and radius $r$, moving at a velocity $\boldsymbol{u}$ is defined from the Helmholtz theorem,

$$
\boldsymbol{A}=\frac{\mu_{0}}{4 \pi} \int_{V} \frac{\boldsymbol{J}}{r} d V=\frac{1}{4 \pi c^{2} \varepsilon_{0}} \int_{V} \frac{\rho \boldsymbol{u}}{r} d V=\frac{\boldsymbol{u}}{c^{2}} \int_{V} \frac{d q}{4 \pi \varepsilon_{0} r}=\frac{\boldsymbol{u}}{c^{2}} \phi_{E}
$$

where $\boldsymbol{J}$ is the current density, $\rho$ is the charge density, $\phi_{E}$ is the electrical scalar-potential and $\mu_{0} \varepsilon_{0}=1 / c^{2}$. The electromagnetic momentum $\boldsymbol{p}_{q}$ of a particle with charge $q$ and velocity $\boldsymbol{u}$ is defined as:

$$
\boldsymbol{p}_{q}=q \boldsymbol{A}=q\left(\frac{\boldsymbol{u}}{c^{2}} \phi_{E}\right)
$$

As the radius of the charge particle approaches to zero, the corresponding scalar-potential and the vector-potential become infinite and give rise to unbounded quantities. However, by using the postulate presented in this paper, the vector-potential and the electromagnetic momentum obtained in terms of scalar potential in equation 13 and 14 become bounded from equation 8 : 
$\boldsymbol{A}=\frac{\boldsymbol{u}}{c^{2}} V_{\text {planck }}$

$\boldsymbol{p}_{q}=\frac{q \boldsymbol{u}}{c^{2}} V_{\text {planck }}$

and equations 12 reads:

$\frac{d E}{d P}=u=\frac{p_{q} c^{2}}{q V_{\text {planck }}}$

The momentum and energy formulas leading to relativistic energy-momentum relations for mass particle and charge particle are tabulated in Table 1. The constant of integration were obtained by introducing rest frame energies, $m_{0} c^{2}$ and $q V_{\text {planck }}$ when the mass and charge particles are at rest respectively.

Table 1. Momentum and energy formulas of mass and charge particles.

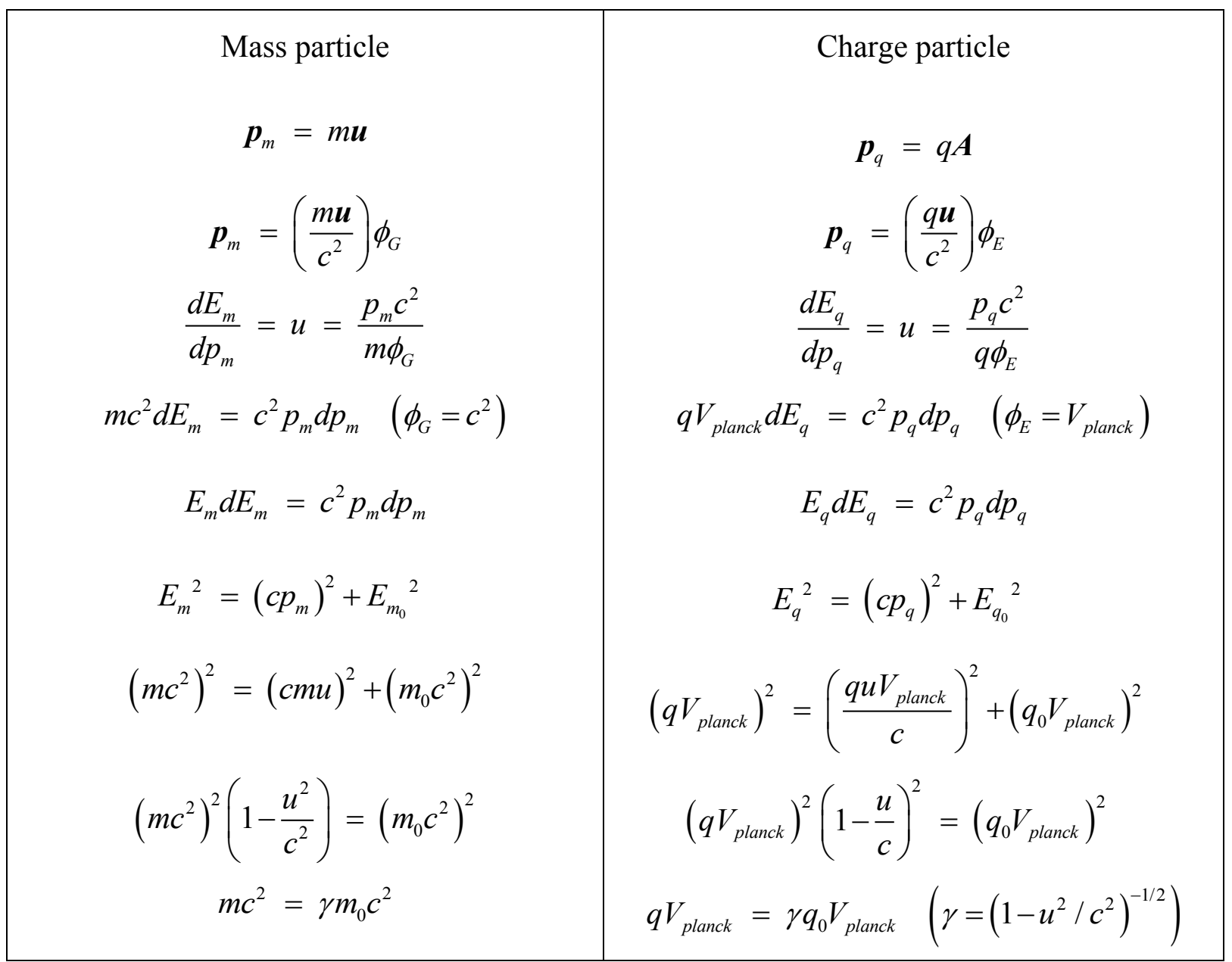


Thereby the energy of a charge particle in motion is relativistically covariant, similar to that of a mass particle in motion. This shows that the mass-energy equivalence and the charge-energy equivalence are both relativistically covariant.

$$
m c^{2}=\gamma m_{0} c^{2} \Rightarrow E_{m}=\gamma E_{m_{0}} \quad \text { and } \quad q V_{\text {planck }}=\gamma q_{0} V_{\text {planck }} \Rightarrow E_{q}=\gamma E_{q_{0}}
$$

From equation 18 ,

$$
m \boldsymbol{u}=\gamma m_{0} \boldsymbol{u} \quad \text { and } \quad q\left(\frac{\boldsymbol{u} V_{\text {planck }}}{c^{2}}\right)=\gamma q_{0}\left(\frac{\boldsymbol{u} V_{\text {planck }}}{c^{2}}\right)
$$

Since the relativistic-mass is derived from the relativistic energy or relativistic momentum of the system, the relativistic-mass $\gamma m_{0}$ is not a good concept. Einstein wrote, "It is not good to introduce the concept of the mass $M=\gamma m_{0}$ of a moving body for which no clear definition can be given. It is better to introduce no other mass concept than the rest-mass $m_{0}$. Instead of introducing $M$, it is better to mention the expressions for the momentum and energy of a body in motion" [17]. The same set of arguments holds true for the relativistic charge $\gamma q_{0}$ associated with relativistic charge-energy $\left(\gamma q_{0} V_{\text {planck }}\right)$ and relativistic charge-momentum

$\left(\gamma q_{0} \boldsymbol{u} V_{\text {planck }} / c^{2}\right)$

expressions as well.

\section{CHARGE PARTICLE AND DE BROGLIE'S MATTER WAVE HYPOTHESIS}

This distinction between waves, propagating according to Maxwell's equations and particles considered to consist of localized particles was challenged when Einstein [18] in 1905 , introduced the concept that light behaved as a collection of localized energy packets or energy quant which was later termed 'photons'. de-Broglie expanded Einstein's hypothesis to all matter particles arguing that just as light exhibits a wave-particle duality, all particles must also be associated with a wave into which they are incorporated [19] and showed that every particle of matter with mass $m$ and velocity $u$, a real wave with a wavelength known as deBroglie's wavelength associated with its momentum exist. That is,

$$
\lambda=\frac{h}{p}=\frac{h}{\gamma m_{0} u} .
$$

Using the concept of de-Broglie, the charge particles can also be associated with a wave into which they are incorporated and their de-Broglie's wavelength for a charge particle with charge $q$ and velocity $u$ is given by

$$
\lambda_{q}=\frac{h}{p_{q}}=\frac{h c^{2}}{\gamma q_{0} u V_{\text {planck }}}
$$


and, the frequency of the matter-wave associated with the charge particle reads:

$$
f_{q}=\frac{h}{E}=\frac{h}{q_{0} \gamma V_{\text {planck }}} \text {. }
$$

In 1927, Davisson and Germer confirmed that electrons diffracted and behaved like waves and the diffraction was thought to have arisen from the electromagnetic mass of electrons [20]. However, the matter-wave relations presented in equations 21 and 22 for a charge particle in motion are derived from a relativistically covariant energy-momentum relation, and therefore could potentially account for the observed scattering of the electrons which give rise to a diffracted wave-like behavior.

\section{RELATIVISTICALLY COVARIANT ENERGY-MOMENTUM FOUR VECTOR}

Max Abraham [21] and H.A Lorentz [22], based on Maxwell's theory of electricity and magnetism developed the first set of theories for the classical electron. From classical electrostatics, the rest energy $U_{0}$ of a spherical charge body with total charge $q$, uniformly distributed over its spherical surface of radius $r$ is given in equation 1 . The relativistic electromagnetic energy $U$ of the charge $q$, moving with velocity $\boldsymbol{u}$ can be derived, similar to derivations given by Panofsky [23]:

$$
U=\frac{1}{2} \int_{V}\left(\varepsilon_{0} E^{2}+\frac{H^{2}}{\mu_{0}}\right) d V=\gamma \frac{q^{2}}{8 \pi \varepsilon_{0} r}\left(1+\frac{u^{2}}{3 c^{2}}\right)
$$

and its relativistic electromagnetic momentum $\mathrm{P}$ as:

$$
\boldsymbol{p}=\varepsilon_{0} \int_{V}(\boldsymbol{E} \times \boldsymbol{B}) d V=\frac{4}{3} \gamma\left(\frac{q^{2}}{8 \pi \varepsilon_{0} r c^{2}}\right) \boldsymbol{u} .
$$

However, according to mass-energy equivalence and the theory of relativity, the equivalent electromagnetic invariant mass $m_{e}$ of an electron with charge $e$ is from equation 1 with $q=e$,

$$
m_{e}=\frac{U_{0}}{c^{2}}=\frac{e^{2}}{8 \pi \varepsilon_{0} r c^{2}} .
$$

The relativistic electromagnetic energy and momentum given in equations 23 and 24 can be written in terms of the electromagnetic invariant mass $m_{e}$ as:

$$
\begin{aligned}
U & =\gamma m_{e} c^{2}\left(1+\frac{u^{2}}{3 c^{2}}\right) \\
\boldsymbol{p} & =\frac{4}{3} \gamma m_{e} \boldsymbol{u} .
\end{aligned}
$$


Therefore relativistic electromagnetic energy and momentum are neither relativistically covariant nor transformed as an energy-momentum four-vector.

From the proposed hypothesis, the electromagnetic invariant mass $m_{e}$ of an electron with charge $e$ can be obtained as,

$m_{e}=\frac{U_{0}}{c^{2}}=\frac{e V_{\text {planck }}}{c^{2}}$

The expressions of the relativistic electromagnetic energy and the momentum obtained in equations 18 and 19 can be expressed in terms of the electromagnetic invariant mass $m_{e}$,

$$
\begin{aligned}
& U=\gamma e V_{\text {planck }}=\gamma m_{e} c^{2} \\
& \boldsymbol{p}=\gamma e\left(\frac{\boldsymbol{u} V_{\text {planck }}}{c^{2}}\right)=\gamma m_{e} \boldsymbol{u}
\end{aligned}
$$

The relativistic electromagnetic energy and the momentum obtained in equations 29 and 30 are relativistically covariant and they form a relativistically covariant energymomentum four-vector in Minkowskian spacetime:

$$
P^{\mu}=\left(\frac{E}{c}, \boldsymbol{p}\right)=\left(\gamma e \frac{V_{\text {planck }}}{c}, \gamma e \frac{V_{\text {planck }}}{c^{2}} \boldsymbol{u}\right)
$$

which gives rise to the energy-momentum relation as shown below.

$$
\begin{aligned}
& P^{\mu} P_{\mu}=\left(\gamma e \frac{V_{\text {planck }}}{c}, \gamma e \frac{V_{\text {planck }}}{c^{2}} \boldsymbol{u}\right)\left(\gamma e \frac{V_{\text {planck }}}{c},-\gamma e \frac{V_{\text {planck }}}{c^{2}} \boldsymbol{u}\right)=\left(\gamma e \frac{V_{\text {planck }}}{c}\right)^{2}\left(1-\frac{u^{2}}{c^{2}}\right)=\left(e \frac{V_{\text {planck }}}{c}\right)^{2} \\
& \left(\gamma e V_{\text {planck }}\right)^{2}=\left(e V_{\text {planck }}\right)^{2}+\left(\gamma e V_{\text {planck }} \frac{u}{c}\right)^{2} \Leftrightarrow U^{2}=U_{0}^{2}+(p c)^{2}
\end{aligned}
$$

\section{STABILITY PROBLEM IN FINITE RADIUS CHARGE PARTICLE MODEL}

Charge particle models with finite radii cannot explain how the repulsive like-charge distributions are held together. In order to explain this stability problem, Poincare, in 1905 introduced a postulate, that a non-electromagnetic force was required, to hold the like-charge distribution together. However, in view of the energy-momentum expression for a collection of mass particles given in equation 5, which quantifies, the total invariant energy of the system without taking into account the potential energies associated with particle interactions, we emphasize that the electromagnetic energy-momentum relation alone would quantify the total energy of a system comprising of many charge particles, without having to incorporate the potential energies arising between its constituent particles. That is, the composite energy 
of the system, associated with the energy-momentum equation, includes both the kinetic energy and the potential energy of the system. This yields that the electromagnetic energymomentum relation obtained for a charge particle in motion can be extended to a collection of many charge particles, and obtain the total invariant charge $Q$ as given below.

$$
\left(Q V_{\text {planck }}\right)^{2}=\left(\sum_{i=1}^{n} E_{i}\right)^{2}-\left|\left(\sum_{i=1}^{n} \frac{\gamma q_{i} V_{\text {planck }}}{c} \boldsymbol{u}_{i}\right)\right|^{2}
$$

Further, if the total energy of a collection of infinitesimal charge quantities can be quantified by the expression given in equation 33, then the momentum energy components associated with constituent charge quantities can be shown to give rise to a set of attractive magnetic forces between themselves, if they are associated with a relative motion in the same sense of direction, which could potentially resolve the stability problem associated with finite radius models. This yields that like-charge particles, such as electrons, with a finite radius model, requires that their corresponding charge distributions to be associated with an intrinsic spin. Thus, the electron model presented in this paper demands for an intrinsic spin associated with the electron's charge distribution. Thus, a theoretical explanation to the electron's spin is obtained.

\section{CONCLUSION}

A new postulate to treat the electric and gravitational scalar-potentials, so that they become finite and bounded was proposed. This led to the derivation of both the chargeenergy and mass-energy equivalences. Extending this postulate to a charge particle in motion, its corresponding energy-momentum equation was derived and the total electromagnetic energy and the electromagnetic momentum associated with a charge particle in motion were relativistically covariant. Using the concept de-Broglie's matter-wave hypothesis deBroglie's wavelength for a charge particle is presented. The stability-problem associated with repulsive like-charge distribution is potentially resolved which demands that the electrons are associated with an intrinsic spin, consistent with the quantum mechanical description of the elementary particles. The present paper is a call for a revision of the classical electrodynamics to make it a fully covariant system with the rest of the classical physics.

\section{Acknowledgement}

The first author is deeply indebted to J.A Gunawardena, whose suggestions and continuous encouragement helped him immensely to investigate and further refine the work presented in this paper.

\section{References}

[1] G. G. Stokes, Trans. Cam. Phil. Soc. 8 (1844) 105-137.

[2] J. J. Thomson, Phil. Mag. 11 (1881) 229-249. 
[3] G. F. C Searle, Phil. Mag. 44 (269) (1897) 329-341.

[4] H. A Lorentz, Proc. Roy. Neth. Acad. Arts and Sci. 6 (1904) 809-831.

[5] H. Poincare, Comptes Rendus 140 (1905) 1504-1508.

[6] E. Fermi, Phys. Zeits. 23 (1922) 340-344.

[7] H. Mandel, Z. Physik 39 (1926) 40.

[8] W. Wilson, Proc. Phys. Soc. (London) 48 (1936) 736-740.

[9] P. A. M. Dirac. Proc. Roy. Soc. (London) A167 (1938) 148-169.

[10] M.H.L. Pryce, Proc. Roy. Soc. (London) A168 (1938) 389-401.

[11] B. Kwal, J. Phys. Radium 10 (1949)103-104.

[12] F. Rohrlich, American Journal of Physics 28 (1960) 639-643.

[13] H. R. Noble, F. T. Trouton, Phil. Trans. Roy. Soc. London A202 (1903)165.

[14] J. W Butler, American Journal of Physics 36 (1968) 936-941.

[15] J. A Stratton, Electromagnetic Theory (Mcgraw Hill,1941) p.

[16] W. Pauli, Thoery of Relativity, (Pergamon Press, 1958) p.

[17] V. Hnizdo, Am. J. Phys. 65 (1997) 55-65.

[18] A. Einstein, Annalen der Physik 18 (1905) 639-643.

[16] E. Hecht, Am. J. Phys. 77 (2009) 799-806. Am. J. Phys. 79 (2011) 591-600.

[17] L.B. Okun, Physics Today, 42 (1989) 31-36.

[18] A. Einstein, Ann. Phys. 17 (1905)132-148.

[19] L de Broglie, Ann. Phys. 3 (1925) 22.

[20] C. D Anderson, Phy. Rev., 43 (1933) 491-494.

[21] M. Abraham, A. Föppl, Theorie der Elektrizitt (Leipzig Teubner, 1905).

[22] H. A Lorentz, Archives nerlandaises des sciences exactes et naturelles 25 (1892) 363-552.

[23] W. K. H Panofsky, M. Phillips, Classical Electricity and Magnetism (Addison-Wesley, 1962). 